\title{
"Falácia da Conjunção": Definição e Variáveis de Controle ${ }^{1}$
}

\author{
Lílian Cavalheiro Rodrigues ${ }^{2}$ \\ The Ohio State University \\ Josele Abreu-Rodrigues \\ Universidade de Brasília
}

\begin{abstract}
RESUMO - A falácia da conjunção ocorre quando a conjunção de dois eventos é julgada como mais provável de ocorrer do que seus eventos constituintes. Esse fenômeno tem sido investigado principalmente por psicólogos cognitivistas, mas, recentemente, tem atraído o interesse de alguns analistas do comportamento. O presente trabalho consiste em um resumo da literatura sobre falácia, no qual são enfatizadas as estratégias metodológicas utilizadas e os resultados empíricos obtidos. Dentre as variáveis controladoras analisadas, são destacadas a representatividade, o uso inadequado de regras probabilísticas, as experiências passadas com os eventos constituintes e compostos, o treino estatístico e os aspectos verbais e contextuais.
\end{abstract}

Palavras-chave: falácia da conjunção; julgamentos de probabilidade; análise do comportamento.

\section{“Conjunction Fallacy": Definition and Control Variables}

\begin{abstract}
The conjunction fallacy occurs when the conjunction of two events is judged as more probable to occur than its constituent events. This phenomenon has been investigated mainly by cognitive psychologists but, recently, it has called the attention of some behavior analysts. The present study summarizes the literature on fallacy, emphasizing the methodological strategies used and the empirical results attained. Among the control variables analyzed, some special attention is given to the representativeness, the inadequate use of probabilistic rules, the previous experiences with constituent and compound events, the statistical training, and verbal and contextual aspects.
\end{abstract}

Key words: conjunction fallacy; probability judgments; behavior analysis.

Um erro de julgamento comumente observado em situações de escolha e tomada de decisão é denominado falácia da conjunção, a qual é observada quando a conjunção de dois eventos é avaliada como mais provável de ocorrer do que um desses eventos sozinho. A falácia da conjunção foi inicialmente relatada por Tversky e Kahneman (1983). Em seu exemplo mais conhecido, os participantes receberam o clássico "problema da Linda": "Linda tem 31 anos, é solteira, extrovertida e muito inteligente. Ela é formada em filosofia. Como estudante, ela era bastante preocupada com questões de discriminação e justiça social e também participou de demonstrações antinucleares" (p. 297). Em seguida, os participantes deveriam julgar a probabilidade de cada uma das seguintes alternativas: "(A) Linda é ativista do movimento feminista; (B) Linda é caixa de banco; $e$ ( $A \& B)$ Linda é caixa de banco e ativista do movimento feminista" (Tversky \& Kahneman, 1983, p. 297).

Foi observado que $85 \%$ dos participantes julgaram o evento constituinte A como mais provável que o composto A\&B e este como mais provável que o evento constituinte B. Esse resultado consiste em um erro de julgamento porque viola um

1 Parte do presente trabalho foi derivada da Dissertação de Mestrado defendida pela primeira autora no Instituto de Psicologia da Universidade de Brasília.

2 Endereço: The Ohio State University, Physical Activities and Educational Services, Columbus, OH, USA 43210-1120.E-mail: liliancrod@ bol.com.br. princípio fundamental da probabilidade, a regra da inclusão: se $\mathrm{X}$ inclui $\mathrm{Y}$, então a probabilidade de $\mathrm{Y}$ não pode ser maior do que a probabilidade de X. Ao ser estendida para eventos compostos, essa regra é denominada "regra da conjunção", a qual estabelece que, no exemplo acima, a probabilidade de $\mathrm{A} \& \mathrm{~B}$ deve ser menor ou igual à probabilidade de $\mathrm{B}$ uma vez que $\mathrm{A} \& \mathrm{~B}$ é um subconjunto de $\mathrm{B}$.

O presente trabalho tem como objetivo introduzir o leitor à literatura sobre falácia da conjunção. Torna-se relevante discutir o fenômeno, considerando que: a) esse fenômeno indica que, quando confrontados com eventos conflitantes, os indivíduos julgam erroneamente as informações disponíveis, b) julgamentos da probabilidade dos eventos são freqüientemente solicitados em contextos educacionais e profissionais, e c) tais erros de julgamento são comumente cometidos até mesmo por indivíduos com treino formal em lógica e teorias de tomada de decisão. Dessa forma, o foco deste texto consiste na identificação e discussão dos determinantes ambientais da falácia, sendo destacadas as metodologias utilizadas e os resultados empíricos obtidos nos estudos analisados. Dentre os determinantes a serem abordados encontram-se a representatividade e a probabilidade dos eventos, a história passada, o treino estatístico, e aqueles relacionados a estímulos verbais e contextuais.

\section{Representatividade Vs. Probabilidade}

Diversos estudos sobre a falácia da conjunção têm investigado duas variáveis de controle: a) a representatividade e b) 
as regras estatísticas e leis de probabilidade (e.g., Gavanski \& Roskos-Ewoldsen, 1991; Stolarz-Fantino, Fantino \& Kulik, 1996; Tversky \& Kahneman, 1983; Wolford, Taylor \& Beck, 1990). Os julgamentos intuitivos da probabilidade de um evento composto são baseados em heurísticas da representatividade, as quais negligenciam considerações outras que não aquelas relacionadas com a similaridade entre um exemplo e um modelo. Isto é, a avaliação da probabilidade do evento composto "Linda é caixa de banco e ativista do movimento feminista" é controlada não pela regra da conjunção, mas sim, pela similaridade entre as informações fornecidas sobre Linda (exemplo) e os estereótipos de caixa de banco e de ativista do movimento feminista (modelos). Dessa forma, o evento composto é considerado mais provável do que o evento simples porque Linda é considerada como mais representativa de "caixas de banco feministas" do que de "caixas de banco". Os julgamentos lógicos da probabilidade de um evento composto, por outro lado, se baseiam em heurísticas estatísticas, as quais consideram as propriedades inclusivas dos eventos relevantes, isto é, seguem a regra da conjunção. Dessa forma, uma vez que o evento simples "caixa de banco" inclui o evento composto "caixa de banco e ativista do movimento feminista", então a probabilidade do evento composto não pode ser logicamente maior do que a probabilidade do evento simples.

\section{Representatividade}

A heurística da representatividade se refere ao "grau de correspondência entre uma amostra e uma população, uma instância e uma categoria, um ato e um ator ou, mais genericamente, entre um exemplo e um modelo" (Tversky \& Kahneman, 1983, p. 295). Segundo esses autores, um exemplo pode ser considerado representativo de um modelo não somente em função da similaridade entre exemplo e modelo, mas também da freqüência de ocorrência do exemplo ou da relação condicional entre exemplo e modelo.

\section{Similaridade}

Um aspecto que torna um exemplo representativo de um modelo é a existência de características comuns em ambos. Por exemplo, um violão é um objeto representativo dos instrumentos de corda porque compartilha uma característica definidora da classe, ou seja, possui cordas. Nesse caso, diz-se que a representatividade ocorre por similaridade ou generalização.

O papel da similaridade na falácia da conjunção foi investigado por Shafir, Smith e Osherson (1990). Nesse estudo, os participantes receberam 14 problemas comparáveis ao problema da Linda, com uma diferença: além de terem que julgar a probabilidade ("Qual a probabilidade de que cada alternativa seja verdadeira?") e a representatividade ("Quão representativa a pessoa descrita no problema é de cada alternativa?”) com relação a itens incompatíveis (e.g., caixa de banco e feminista vs. caixa de banco), eles tinham que fazer o mesmo com relação a itens compatíveis (e.g., socióloga e feminista vs. socióloga). A conjunção "caixa de banco e feminista" é incompatível porque caixas de banco e feministas têm poucas propriedades em comum; por outro lado, "socióloga e feminista" é uma conjunção compatível porque feministas típicas compartilham muitas propriedades com sociólogas típicas. Os participantes julgaram a alternativa composta, tanto compatível quanto incompatível, como mais provável do que seu constituinte menos provável, além de julgarem a pessoa descrita em cada problema como mais representativa da alternativa composta do que de seus constituintes. Entretanto, a incidência (número de ocorrências) e a magnitude (o valor estimado para o composto em comparação ao evento constituinte de menor valor) dos julgamentos da probabilidade foram maiores para as conjunções incompatíveis do que para as conjunções compatíveis, o mesmo ocorrendo com os julgamentos da representatividade (ver também Wells, 1985). Além disso, foi encontrada uma correlação positiva entre a incidência (e a magnitude) de ambos os julgamentos, o que levou os autores a concluírem que julgamentos de probabilidade são uma função crescente dos julgamentos de representatividade.

\section{Freqüência}

A representatividade também é afetada pela freqüência com que o evento ocorre, uma vez que eventos mais freqüentes tendem a ser considerados mais representativos. Por exemplo, problemas cardiovasculares são mais representativos de indivíduos acima de 50 anos do que daqueles entre 20 e 30 anos já que a incidência desses problemas é maior entre indivíduos mais velhos.

Tversky e Kahneman (1983) apresentaram um exemplo do controle exercido pela freqüência dos eventos. No experimento, os participantes receberam a seguinte instrução (p. 303):

Considere um dado com seis faces (quatro faces verdes e duas
vermelhas). O dado será jogado 20 vezes e as seqüencias de
verdes $(V d)$ e vermelhos (Vm) serão registradas. Selecione uma
seqüência, dentre as três possíveis, e você ganhará $\$ 25$ se a
seqüência que você escolher aparecer em sucessivas jogadas
do dado. Por favor, marque a seqüência de verdes e vermelhos
na qual você prefere apostar.
1) Vm-Vd-Vm-Vm-Vm
2) $V d-V m-V d-V m-V m-V m$
3) $V d-V m-V m-V m-V m-V m$

Note que a Seqüência 1 pode ser obtida a partir da Seqüência 2, bastando excluir o primeiro Vd. Dessa forma, a Sequiência 1 é mais provável de ocorrer do que a Sequiência 2 , pois sempre que esta última ocorrer, a primeira também ocorrerá, mas o inverso não é verdadeiro. Note também que as seqüências 1 e 3 são menos representativas do dado do que a Seqüência 2, pois essa última contém uma proporção maior da cor mais freqüente $(\mathrm{Vd})$. Os resultados indicaram que aproximadamente $60 \%$ dos participantes escolheram apostar na Sequiência 2, a mais representativa, em detrimento da Sequiência 1, a mais provável.

Contudo, a covariação entre frequiência e representatividade não é necessariamente observada, pois um exemplo específico pode ser bastante representativo de um modelo mesmo sendo infreqüente. Tversky e Kahneman (1983), por exemplo, relataram que $68 \%$ dos participantes de um 
grupo de estudantes universitários afirmaram que era mais representativo de uma aluna da universidade pesar $57 \mathrm{Kg}$ do que mais de $62 \mathrm{Kg}$, enquanto $78 \%$ dos participantes de outro grupo disseram que alunas com mais de $62 \mathrm{Kg}$ eram mais freqüentes na universidade do que alunas com $57 \mathrm{Kg}$. Esses resultados mostram que julgamentos baseados na heurística da representatividade, além de não serem limitados pelas regras de inclusão entre conjuntos, não são determinados, necessariamente, pela freqüência do evento.

\section{Relações condicionais}

Representatividade pode também se referir à propensão do modelo a produzir o exemplo, isto é, à existência de uma história causal entre os eventos. Por exemplo, o isolamento social é considerado uma ação representativa da depressão porque se atribui às pessoas depressivas a disposição de evitar o convívio social.

No problema padrão da Linda, a falácia ocorre mesmo não havendo relação causal ou correlacional entre os eventos constituintes (feminista e caixa de banco). Tversky e Kahneman (1983) sugeriram, entretanto, que se houver uma relação condicional entre esses eventos (e.g., ter pós-graduação em Administração e ser caixa de banco), a incidência da falácia deveria aumentar, sendo esse aumento diretamente proporcional à magnitude dessa relação condicional. Essas sugestões foram avaliadas por Fabre, Caverni e Jungermann (1995, Experimento 2). Nesse estudo, os participantes receberam quatro textos, cada texto com descrições de duas doenças. Essas doenças poderiam ou não ser causalmente relacionadas e, quando havia uma relação causal, esta poderia apresentar uma magnitude alta ou baixa. Por exemplo, um dos textos descrevia duas doenças: o reumatismo degenerativo e a anemia. Na versão causal, a descrição do reumatismo indicava que o tratamento produzia efeitos colaterais, entre eles, uma diminuição da vitamina B12 no organismo. No caso de magnitude alta, era dito que esse efeito colateral era freqüente, enquanto no caso de magnitude baixa, era dito que esse efeito era apenas possível. A descrição da anemia dizia que a causa primária da doença era a falta de vitamina B12. Na versão não-causal, nenhum dos efeitos da primeira doença era apontado como causa potencial da segunda doença. Em seguida, os participantes tinham que julgar a probabilidade de uma determinada pessoa ter a primeira doença, ter a segunda doença e ter ambas as doenças. Os resultados mostraram que a incidência e magnitude da falácia foram maiores na presença do que na ausência de relação causal entre as doenças, mas não foram afetadas pela magnitude dessa relação, confirmando apenas parcialmente as sugestões de Tversky e Kahneman (1983). Resultados similares foram obtidos por Fisk e Pidgeon (1998).

Relações causais e correlacionais entre eventos constituintes também afetam diferencialmente a magnitude da falácia. Por exemplo, Ahn e Bailenson (1996) observaram que a magnitude da falácia foi mais alta com explicações causais (e.g., "Kim teve um acidente de carro porque ela tende a não usar óculos enquanto dirige, apesar de ser míope") do que com explicações correlacionais (e.g., "Kim teve um acidente de carro porque é muito mais provável que ela tenha acidentes do que outras pessoas"), possivelmente porque as primeiras foram tidas como mais representativas do que as últimas (ver também Leddo, Abelson \& Gross, 1984; McClure, Lalljee, Jaspars \& Abelson, 1989).

Evidências adicionais do controle pela representatividade foram fornecidas por Stolarz-Fantino e cols. (1996, Experimentos 1 e 4). Esses autores investigaram se a presença ou ausência de informações sobre o indivíduo no enunciado do problema influenciaria a ocorrência da falácia. Um grupo de estudantes universitários recebeu o problema padrão da Linda (com informações completas); o outro grupo recebeu o mesmo problema, mas o enunciado continha apenas as seguintes afirmativas: "Linda tem 34 anos. Você não sabe nada mais sobre ela". Dentre os participantes do grupo com informação, $78 \%$ cometeram a falácia e, dentre aqueles do grupo sem informação, apenas $41 \%$ cometeram a falácia. Stolarz-Fantino, Fantino, Zizzo, e Wen (2003, Experimento 3) também obtiveram resultados similares. Esses estudos mostram que a presença de informações sobre o indivíduo é uma variável crítica para a ocorrência da falácia, provavelmente porque possibilita ao participante avaliar a representatividade dos eventos a serem julgados. $\mathrm{O}$ fato da ausência do enunciado não ter eliminado totalmente a falácia em nenhum desses dois experimentos, entretanto, sugere a existência de outras variáveis de controle além da representatividade. A probabilidade dos eventos constituintes está entre essas possíveis variáveis e será discutida a seguir.

\section{Probabilidade}

Gavanski e Roskos-Ewoldsen (1991) demonstraram que a falácia da conjunção não pode ser explicada unicamente com base na heurística da representatividade. Neste estudo, os participantes foram distribuídos em três grupos, cada um exposto a um tipo específico de problema. O primeiro tipo, denominado de "padrão", compreendia problemas similares ao da Linda. Em cada um deles (e.g., "Linda é caixa de banco e ativista do movimento feminista"; "Bill é contador e toca jazz como hobby"), era possível julgar, diretamente, os eventos constituintes e o composto, a partir da representatividade dos mesmos. O segundo tipo de problema era chamado de "misto". Os mesmos problemas-padrão eram apresentados, mas o composto era formado por um evento constituinte de dois problemas diferentes (e.g., "Linda é caixa de banco" e "Bill é um contador"). Os problemas mistos permitiam que os participantes julgassem a probabilidade dos eventos constituintes sob o controle da representatividade, mas o mesmo não poderia ocorrer para o composto, uma vez que este continha eventos relacionados a diferentes problemas (i.e., a diferentes indivíduos). Assim sendo, o composto só poderia ser avaliado com base na combinação das probabilidades dos eventos constituintes. O terceiro tipo de problema era chamado de "combinação de probabilidade". Nele eram fornecidas as probabilidades de que uma pessoa imaginária tivesse as características A e B (eventos constituintes), devendo o participante estimar a probabilidade dessa pessoa ter as duas características (A\&B), mesmo sem saber mais nada a seu respeito. Esse problema não permitia que os julgamentos da probabilidade dos eventos constituintes e da conjunção fossem controlados pela representatividade, uma vez que não 
havia descrições dos indivíduos, só sendo possível o controle pelas probabilidades dos eventos constituintes.

Os julgamentos da probabilidade do composto não diferiram significativamente nos três grupos investigados. Considerando que esses julgamentos não poderiam ser efetuados a partir da representatividade em dois dos três grupos (problemas "misto" e "combinação de probabilidade"), mas que poderiam ser efetuados com base nas probabilidades dos eventos constituintes em todos os três grupos, Gavanski e Roskos-Ewoldsen (1991) concluíram que é possível que a representatividade esteja relacionada com a ocorrência da falácia porque afeta o julgamento da probabilidade dos eventos constituintes, mas que o julgamento do composto é feito com base na combinação da probabilidade desses eventos. Isso ocorreria porque, embora os indivíduos tenham estereótipos para "caixas de banco" e para "feministas", por exemplo, eles normalmente não têm estereótipos para "caixas de banco e feministas". Na ausência da possibilidade de controle pela representatividade, a probabilidade dos eventos constituintes assumiria o controle dos julgamentos do composto. Entretanto, esse controle não é necessariamente consistente com as leis da probabilidade, ou seja, as probabilidades dos eventos constituintes nem sempre são combinadas corretamente, ocasionando, assim, a falácia da conjunção.

\section{Regras de probabilidade}

Duas regras incorretas de combinação de probabilidades têm sido comumente apontadas como explicação para a ocorrência da falácia: soma e média. Nos dois casos, a identificação da regra que controlou o julgamento da probabilidade do composto é baseada na comparação entre as probabilidades do composto e de seus constituintes. Controle pela soma das probabilidades dos constituintes é inferido quando a probabilidade estimada do composto é maior do que a probabilidade de ambos os eventos constituintes (e.g., Fantino \& Savastano, 1996; Gavanski \& Roskos-Ewoldsen, 1991; Rodrigues, 2005). Por outro lado, controle pela média das probabilidades dos constituintes é sugerido quando a probabilidade estimada do composto é maior do que a probabilidade do evento constituinte menos provável e menor do que aquela do evento constituinte mais provável (e.g., Fantino, Kulik, Stolarz-Fantino \& Wright, 1997; Gavanski \& Roskos-Ewoldsen, 1991; Shanteau, 1975; Zizzo, StolarzFantino, Wen \& Fantino, 2000).

No estudo de Gavanski e Roskos-Ewoldsen (1991), por exemplo, os julgamentos de probabilidade do composto e os relatos pós-experimentais mostraram que, embora o cálculo da média dos eventos constituintes tenha sido a estratégia prioritariamente utilizada para combinar as probabilidades dos eventos constituintes, também ocorreu controle pela soma das probabilidades. Evidência adicional de controle dos julgamentos do composto pela média das probabilidades foi fornecida por Fantino e cols. (1997). Os autores testaram estudantes universitários em problemas similares ao da Linda e observaram que quanto maior a discrepância entre os valores das probabilidades dos eventos constituintes, mais freqüente a ocorrência da falácia, um resultado compatível com o que seria esperado caso os julgamentos do composto tivessem sido controlados pelo valor médio das probabilidades dos eventos constituintes (ver também, Anderson, 1965; Massaro, 1994; Stolarz-Fantino \& cols., 2003, Experimento 4; Tversky \& Kahneman, 1983). Isso porque, quando o composto é formado por eventos com probabilidades divergentes (e.g., $0,2$ e 0,8$)$, a média das probabilidades $(0,5)$ é claramente superior à probabilidade de ocorrência do evento constituinte de menor probabilidade $(0,2)$, um fato que não ocorre com eventos com probabilidades similares (e.g., 0,2 e 0,3 ), o que deveria, então, ocasionar uma maior incidência de falácia com eventos discrepantes.

\section{Magnitude das probabilidades}

Essa variável foi investigada por Gavanski e RoskosEwoldsen (1991). Nesse estudo, os participantes foram divididos em três grupos, os quais diferiam em termos da probabilidade dos eventos que formavam o composto (altaalta, alta-baixa e baixa-baixa). Verificou-se que os valores das probabilidades dos eventos constituintes tiveram efeitos diferenciais sobre a incidência e magnitude da falácia. Com relação à incidência, os participantes tiveram menor tendência a cometer a falácia no caso de conjunções com dois eventos com baixa probabilidade do que no caso de conjunções com dois eventos com alta $v s$. alta ou alta $v s$. baixa probabilidades, confirmando os resultados de Fantino e cols. (1997). Com relação à magnitude da falácia, foi observado que os participantes apresentaram estimativas mais altas quando o composto era constituído por eventos com probabilidades discrepantes do que por eventos com probabilidades similares (ver também Fabre \& cols., 1995).

\section{Tipo de julgamento das probabilidades}

Diversos estudos têm apontado que algumas características dos problemas influenciam o controle exercido pelas probabilidades. Stolarz-Fantino e cols. (1996, Experimento 1) avaliaram se a falácia seria afetada quando 1) os participantes estimam, eles próprios, as probabilidades dos eventos constituintes ou são informados, pelo experimentador, sobre o valor dessas probabilidades, e 2) os participantes não estimam e não recebem os valores das probabilidades, cabendo-lhes apenas marcar um $\mathrm{X}$ na alternativa que julgar como mais provável. A investigação da primeira questão experimental não revelou diferença na incidência de falácia entre o grupo que estimou os valores dos eventos constituintes e o grupo que já recebeu esses valores do experimentador (47\%, no primeiro grupo e $48 \%$, no segundo). $\mathrm{Na}$ investigação da segunda questão experimental, marcar um $\mathrm{X}$ produziu uma incidência de falácia de $72 \%$.

Sloman, Over, Slovak e Stibel (2003, Experimento 5) solicitaram a estudantes universitários que avaliassem as probabilidades de diversos eventos simples e de um evento composto, no problema da Linda, de duas formas distintas: metade dos participantes deveria ordenar os eventos (simples e compostos) do mais para o menos provável enquanto a outra metade deveria apresentar a probabilidade de cada evento. A falácia da conjunção foi menos freqüente quando os participantes tinham que estimar as probabilidades (ver também Zizzo \& cols., 2000, Experimento 2) do que quando tinham de ordenar os eventos. Finalmente, Stolarz-Fantino e cols. 
(2003, Experimento 2) relataram que quando os participantes tinham que estimar a probabilidade do composto antes de estimarem a probabilidade dos eventos constituintes, o que impedia que os julgamentos do composto fossem afetados pelos julgamentos de seus constituintes, a incidência da falácia foi maior (aproximadamente 55\%) do que quando o composto era o último evento a ser julgado (31\%).

Em suma, esses resultados indicam que quando as probabilidades dos eventos constituintes não são explicitamente enfatizadas, o controle por essas probabilidades é enfraquecido, dando margem para que outras variáveis exerçam controle sobre o julgamento.

\section{Variáveis Históricas}

Diversos estudos têm indicado que o comportamento é resultado não somente de contingências atuais, mas também de contingências históricas (e.g., Freeman \& Lattal, 1992; Wanchisen, 1990). Dessa forma, é viável supor que o julgamento da probabilidade do composto e, conseqüentemente, a falácia da conjunção, resultem não somente das contingências experimentais presentes no momento em que esse julgamento é efetuado, mas também da história de reforçamento e punição correlacionada com cada evento constituinte ou, até mesmo, com o evento composto.

Evidências de controle pela história pré-experimental foram fornecidas por Teigen, Martinussen e Lund (1996a). Nesse estudo, os autores avaliaram como uma história favorável (ou desfavorável) tanto a um evento constituinte quanto ao um evento composto afetaria o julgamento de probabilidade do evento composto. No Experimento 2, estudantes universitários estimaram os resultados de um referendum sobre a inclusão de três países na União Européia. Para tanto, metade dos participantes apontou a probabilidade do resultado ser "SIM" e a outra metade, a probabilidade do resultado ser "NÃO", em cada país isoladamente e em dois (e três) países conjuntamente. Além disso, todos os participantes indicaram sua própria opção de voto ("SIM" ou "NÃO"). Dessa forma, o participante poderia ter uma opção de voto "SIM" e apontar uma probabilidade alta para o resultado "SIM" (concordância) ou uma probabilidade alta para o resultado "NÃO" (discordância), o mesmo podendo ocorrer quando a opção de voto era "NÃO". Foi observado que os participantes de ambos os grupos cometeram menos falácia quando avaliaram resultados com os quais discordavam do que com os quais concordavam. Ou seja, ao avaliar a conjunção de um evento indesejável, os participantes emitiram respostas mais aceitáveis do ponto de vista lógico, o que sugere que preferências pessoais (ou, alternativamente, a história passada) afetam a ocorrência de falácia.

Fantino e Savastano (1996), por sua vez, adotaram um procedimento comum na Análise Experimental do Comportamento para investigar o efeito da história de reforçamento correlacionada com eventos compostos sobre a escolha por estímulos compostos novos. Seu procedimento não é análogo àquele comumente utilizado nos estudos de falácia, mas há uma semelhança entre eles: os eventos compostos, em ambos os procedimentos, incluem eventos com alta e baixa probabilidade. Os participantes foram expostos a uma tarefa de escolha de acordo com o modelo em que deveriam escolher, dentre dois estímulos coloridos, aquele idêntico ao modelo. Na primeira sessão, duas cores (vermelho e verde) eram correlacionadas com uma alta probabilidade de reforçamento para escolhas corretas $(p=0,8)$; as outras duas cores (azul e amarelo) eram correlacionadas com uma baixa probabilidade de reforçamento $(p=0,2)$. Na segunda sessão, os participantes foram divididos em dois grupos. Para o Grupo Sem Treino, não houve alteração na condição experimental. Para o Grupo Com Treino, por outro lado, foi feita a seguinte alteração: em 1/5 das tentativas, o modelo consistia em um estímulo composto que compreendia um estímulo correlacionado com alta probabilidade (vermelho) e outro correlacionado com uma baixa probabilidade (azul) de reforço. Quando esse modelo era apresentado, as escolhas corretas eram reforçadas em $10 \%$ das tentativas $(p=0,1)$. Na terceira sessão, todos os participantes foram expostos a tentativas de sondagem, nas quais tinham que escolher, entre duas alternativas, aquela com maior probabilidade de gerar reforços: um estímulo composto novo (verde e amarelo) versus um de seus estímulos constituintes (verde ou amarelo).

Os participantes do Grupo Sem Treino mostraram preferência pelo estímulo composto, o que não foi observado com os participantes do Grupo Com Treino, indicando que a experiência prévia com um estímulo composto correlacionado com uma probabilidade de reforço mais baixa do que aquela correlacionada com cada um de seus eventos constituintes reduziu o controle exercido por estímulos compostos novos. Esse resultado parece sugerir que a falácia da conjunção pode ser eliminada caso seja fornecida uma história de reforçamento apropriada para o indivíduo. Um aspecto adicional a ser considerado é que a metodologia utilizada por Fantino e Savastano (1996) permite investigar o papel da história de reforçamento sobre a escolha por estímulos compostos sem que variáveis outras, tais como a representatividade, estejam presentes.

Os efeitos da história, tanto de reforçamento quanto de punição, correlacionada aos eventos constituintes, foi investigada por Rodrigues (2005) por meio de uma metodologia similar àquela empregada por Fantino e Savastano (1996). Na Fase de Treino, estudantes universitários foram expostos a quatro estímulos simples coloridos que eram correlacionados com diferentes probabilidades de reforçamento $(\mathrm{Sr})$ e punição (Sp): dois estímulos foram correlacionados com alta probabilidade $(0,8)$ e dois com baixa probabilidade $(0,2)$, sendo que um dos estímulos de cada par foi correlacionado com reforçamento (ganho de pontos) e o outro com punição (perda de pontos). Na Fase de Teste, os participantes foram expostos a tentativas de escolha entre 1) estímulos compostos constituídos de estímulos correlacionados somente com reforçamento ( $\mathrm{Sr} 0,8 / \mathrm{Sr} 0,2)$, somente com punição ( $\mathrm{Sp} 0,8 / \mathrm{Sp}$ $0,2)$ ou com reforçamento e punição ( $\mathrm{Sr} 0,8 / \mathrm{Sp} 0,2 ; \mathrm{Sr} 0,2 / \mathrm{Sp}$ $0,8)$ e 2) estímulos simples de alta e baixa probabilidade de reforçamento ( $\mathrm{Sr} 0,8$ e $\mathrm{Sr}$ 0,2, respectivamente) ou punição (Sp 0,8 e Sp 0,2, respectivamente).

Os participantes mostraram preferência pelos compostos quando estes continham apenas estímulos correlacionados com reforçamento e preferência pelo estímulo simples quando os compostos continham apenas estímulos correlacionados com punição. Quando os compostos compreendiam estímulos correlacionados com reforçamento e punição, os 
participantes escolheram a alternativa (composta ou simples) com maior valor reforçador (ou menor valor punitivo) e se esquivaram daquela com menor valor reforçador (ou maior valor punitivo). Por exemplo, quando o composto $\mathrm{Sr} 0,2 / \mathrm{Sp}$ 0,8 foi comparado com estímulos simples correlacionados com reforço, os participantes escolheram os estímulos simples ( $\mathrm{Sr} 0,2$ ou Sr 0,8); quando esse composto foi comparado com os estímulos correlacionados com punição, houve preferência pelo composto quando o estímulo simples era Sp 0,8 , mas não quando era $\mathrm{Sp} 0,2$. Rodrigues (2005) extrapolou esses resultados para o contexto da falácia. Considerando o problema da Linda, a autora sugeriu que é possível que um indivíduo tenha uma história de baixa probabilidade de reforçamento com "feministas" e alta probabilidade de punição com "caixas de banco". Ao ter que julgar a probabilidade dos eventos, "caixa de banco e feminista" poderia ser julgado como menos provável do que "feminista" porque o composto incluiria punição. Similarmente, "caixa de banco e feminista" poderia ser julgado como mais provável do que "caixa de banco" porque o composto incluiria reforçamento (ver também Anderson, 1965).

\section{Treino Estatístico}

Uma vez que a história passada afeta a falácia, uma questão que se segue é se um treino prévio com regras probabilísticas, leis estatísticas e/ou tomada de decisão poderia eliminar a falácia. Fiedler (1988, Experimento 3) forneceu um treino sobre a teoria dos conjuntos para um grupo de estudantes de segundo grau. Durante esse treino, os participantes foram confrontados com representações gráficas de relações entre eventos (i.e., diagramas de Venn) e, em seguida, solicitados a desenhar várias relações de inclusão, disjunção, conjunção e sobreposição entre eventos adicionais. O segundo grupo não recebeu esse treinamento prévio. A comparação entre o percentual de falácia cometido pelos grupos não revelou diferenças (42\% para o grupo com treino e $40 \%$ para o grupo sem treino).

O treino prévio com diagramas de Venn, entretanto, reduziu a incidência de falácia no estudo de Agnoli e Krantz (1989) com 120 mulheres. O treino incluía as relações lógicas de inclusão, disjunção e sobreposição. Os participantes aprendiam que uma categoria é reduzida quando propriedades restritivas são adicionadas à sua definição (e.g., adicionar a característica "20 a 30 anos" à "nascida nos EUA" reduz o tamanho da categoria sensivelmente). O treino também encorajava o participante a pensar sobre a frequiência das categorias. Por exemplo, era apontado que a categoria "Pais de duas crianças em New Jersey" tem uma freqüência maior do que "Homens de New Jersey com ficha criminal". Além disso, durante o treino, os participantes deveriam desenhar as relações entre eventos, usando para tal o diagrama de Venn, e recebiam feedbacks mostrando a representação correta no caso de erros. Os resultados mostraram que o grupo com treino teve uma incidência de falácia menor (por volta de 44\%) do que o grupo sem treino (aproximadamente $73 \%$ ). A inconsistência entre esses resultados e aqueles encontrados por Fiedler (1988) ocorreu, provavelmente, devido a diferenças entre os treinos. Ambos incluíam representações gráficas de diversas relações, contudo, Agnoli e Krantz (1989) enfatiza- ram também a redução do tamanho de uma categoria quando uma segunda categoria é adicionada à primeira e incentivaram os participantes a pensar em termos de freqüência. Por fim, o feedback fornecido por esses últimos autores pode ter sido decisivo na aprendizagem das relações lógicas (ver também Abreu-Rodrigues, Baumann \& Souza, 2005; Crandall \& Greenfield, 1986; Stolarz-Fantino \& cols., 1996, Experimento 3; Stolarz-Fantino \& cols., 2003, Experimento 3),

A falácia também foi reduzida por meio de treinamento no estudo de Moutier e Houdé (2003). Na Fase de Pré-Teste, estudantes universitários franceses foram solicitados a estimar probabilidades em um problema similar ao da Linda. $\mathrm{Na}$ Fase de Treino, os participantes deveriam estimar o número de palavras com sete letras, em um total de 2000 palavras, que tinham os seguintes formatos: " _n _.". Após apresentarem suas estimativas, os participantes foram divididos em três grupos. Para aqueles que receberam o treino de inibição, era enfatizado que 1) a maior familiaridade com o formato "nt" desviava a atenção do formato menos familiar "n", ocasionando erros, sendo, então, necessário deixar o formato "nt" de lado e considerar o outro formato, e 2) o formato "n" incluía o formato "nt". Para aqueles que receberam o treino lógico, era enfatizada somente a relação de inclusão. Um terceiro grupo não recebeu nenhum tipo de treino. Na Fase Pós-Teste, todos os participantes foram novamente expostos ao problema inicialmente utilizado. A comparação dos resultados das fases pré e pós-teste mostrou redução na incidência da falácia, mas apenas para o grupo que recebeu treino de inibição (redução de aproximadamente 50\%).

Os estudos descritos acima mostraram que procedimentos que incentivam o uso de regras estatísticas podem diminuir a ocorrência da falácia, mas não são suficientes para eliminá-la, sugerindo que outras variáveis interferem na sua ocorrência. Dentre essas outras possíveis variáveis, encontram-se as variáveis verbais, apresentadas a seguir.

\section{Variáveis Verbais}

Conforme visto até o momento, violações da regra da conjunção podem ocorrer em função do controle exercido pela representatividade e por regras inadequadas de probabilidade. A literatura, entretanto, tem apontado que a falácia pode ser também ocasionada pelo controle de variáveis verbais estranhas presentes no contexto experimental.

\section{Probabilidade vs. frequiência}

Alguns autores argumentaram que a compreensão errônea da palavra "probabilidade", sempre presente no enunciado dos problemas, poderia contribuir para a ocorrência da falácia (e.g., Fiedler, 1988; Tversky \& Kahneman, 1983). No estudo de Fiedler (1988, Experimento 1), metade dos participantes recebeu a palavra "probabilidade" no enunciado do problema, enquanto a outra metade recebeu a palavra "freqüência". Essa manipulação reduziu a incidência da falácia de $70 \%$ (participantes do grupo "probabilidade") para 20\% (participantes do grupo "frequiência"). Vale ressaltar que as amostras usadas para os julgamentos de freqüência compreendiam sempre 100 indivíduos, o que pode ter contribuído para as diferenças observadas entre os dois tipos de julgamentos. Mais especi- 
ficamente, as pessoas rotineiramente fazem julgamentos de frequiência a partir de uma amostra de 100 casos, ou seja, em termos percentuais, de modo que a maior familiaridade com esse tipo de julgamento pode ter ocasionado a redução na falácia. Para verificar essa suposição, no Experimento 2 foi manipulado o número de indivíduos na amostra a ser julgada nos diferentes problemas (e.g., 168 indivíduos, 539 indivíduos). Novamente, questões sobre probabilidade geraram mais falácia $(70 \%)$ do que questões sobre freqüência (25\%). Esse resultado sugere que a diminuição da falácia, observada com julgamentos de frequiência, não poderia ser atribuída à maior familiaridade com julgamentos percentuais.

É possível, entretanto, que a diferença esteja no fato do conceito de probabilidade ser vago semanticamente e evocar outros significados (e.g., plausibilidade) que não o significado matemático (e.g., porcentagem, frequiência relativa, certeza). Essa possibilidade foi avaliada por Hertwig e Gigerenzer (1999, Experimento 1). Nesse estudo, a ênfase recaiu sobre a correspondência entre os diversos significados da palavra "probabilidade" e a incidência da falácia. Os participantes receberam o problema da Linda e foram instruídos a ordenar os eventos de acordo com suas probabilidades. Em seguida, os participantes eram solicitados a escolher, numa lista com 13 interpretações diferentes da palavra "probabilidade" (matemáticas e não matemáticas), a palavra que melhor descrevia seu entendimento do problema da Linda. Os resultados mostraram que $83 \%$ dos participantes cometeram a falácia. As cinco palavras mais escolhidas (possibilidade, conceptibilidade, plausibilidade, razoabilidade e tipicalidade, nesta ordem) consistiram em interpretações não matemáticas da palavra "probabilidade". Os autores concluíram que, uma vez que os participantes tendem a interpretar o conceito de probabilidade não matematicamente, escolher o composto não seria uma violação da regra da conjunção já que a probabilidade matemática não estaria sendo avaliada.

Em um experimento subseqüente, Hertwig e Gigerenzer (1999, Experimento 3) utilizaram um procedimento similar para avaliar os significados da palavra "frequiência". Aqui os participantes tinham que estimar a frequiência, em vez da probabilidade, dos eventos no problema da Linda. Nenhum dos participantes cometeu a falácia, tendo apresentado, quase que exclusivamente, interpretações matemáticas para o conceito de freqüência (quão freqüentemente, número, porcentagem, proporção e quantidade foram as cinco mais frequientes, nesta ordem).

Sloman e cols. (2003), por outro lado, questionaram a sugestão de que usar o formato de freqüência favorece o cumprimento da regra da conjunção porque a palavra "probabilidade" é polissêmica enquanto o significado da palavra "frequiência" é naturalmente matemático. Assim como Tversky e Kahneman (1983), esses autores argumentaram que a redução na falácia ocorre porque o formato de frequiência torna as relações de inclusão entre os eventos mais transparente. Para avaliar essa possibilidade, os participantes foram expostos a uma situação em que a relação de inclusão era obscurecida pela presença de eventos distrativos entre o evento constituinte e o evento composto. No problema da Linda, por exemplo, foram acrescentadas nove alternativas às três tradicionalmente utilizadas, tais como: é uma professora, ler como hobby, gosta de festas, é uma psicóloga, fuma cigar- ros. Os participantes tinham, então, que ordenar os eventos em termos de suas probabilidades ou de suas frequiências. Não foram encontradas diferenças entre os grupos (aproximadamente $79 \%$ e $75 \%$, respectivamente, de incidência da falácia), o que levou os autores a concluírem que os efeitos positivos do formato de frequiência são eliminados quando a relação entre eventos é ambígua. Resultados comparáveis foram obtidos por Mellers, Hertwig e Kahneman (2001), ou seja, a incidência da falácia foi maior quando o problema, apresentado no formato de freqüência, compreendia estímulos distrativos do que quando compreendia apenas os eventos constituintes e o evento composto.

\section{Conectivos}

Alguns autores têm argumentado que a ocorrência da falácia seria decorrente da interpretação errônea do conectivo "e". Hertwig (citado por Mellers \& cols., 2001), por exemplo, apontou que o conectivo "e" se refere à "interseção" entre eventos na teoria da probabilidade, mas pode significar tanto "união" quanto "interseção" na linguagem cotidiana. Para evitar ambigüidades, Hertwig propôs que o termo "e" (e.g., "caixas de banco $e$ feministas ativas") fosse substituído por "e são" (e.g. "caixas de banco e são feministas ativas") ou por "que são" (e.g., "caixas de banco que são feministas ativas"), o que tornaria a união dos eventos menos provável. Essa sugestão foi investigada por Mellers e cols. (2001, Experimento 2) por meio de problemas apresentados no formato de freqüência. A falácia da conjunção ocorreu na presença do conectivo "e", mas não na presença de "e são" e "que são", um resultado que sugere que a ênfase na interseção entre eventos favorece o seguimento da regra da conjunção (ver também Fisk \& Pidgeon, 1998; Teigen \& cols., 1996a, Experimento 3).

É também possível que a falácia ocorra porque os participantes interpretam o evento constituinte menos provável (B) como sendo "B e não A". Para avaliar essa possibilidade, Agnoli e Krantz (1989) solicitaram aos participantes que ordenassem diversos eventos de acordo com sua probabilidade em sete problemas distintos. Dentre esses eventos encontravam-se um evento composto e um de três outros eventos: "B" (e.g., "Linda é caixa de banco"), "B e não A" (e.g., "Linda é caixa de banco e não é feminista"), e "B e talvez A" (e.g., "Linda é caixa de banco e talvez seja ou não seja feminista"). Se a falácia é ocasionada pela interpretação errônea de B, os grupos "B" e "B e não A" deveriam ter apresentado resultados similares, o que não ocorreu: a magnitude da falácia foi maior para os participantes do grupo "B e não A" do que para o grupo "B" (ver também Bonini, Tentori \& Osherson, 2004). Esse resultado sugere controle pela representatividade uma vez que houve mais falácia quando foi enfatizada a diferença entre o evento constituinte e a descrição do indivíduo (e.g., a descrição de Linda tornou-se mais representativa de "caixa de banco e feminista" quando essa alternativa foi comparada com "caixa de banco e não feminista" do que quando comparada com "caixa de banco"). Além disso, a magnitude da falácia foi um pouco menor para o grupo "B e talvez A" do que para o grupo "B", o que sugere que a frase "B e talvez A" promove o controle por relações de inclusão. Tversky e Kahneman (1983) também observaram 
uma redução da falácia quando substituíram "B" por "B se A ou não A" (e.g., "Linda é uma caixa de banco a despeito de ser ou não ativa no movimento feminista").

\section{Auto-instruções e instruções}

Zizzo (2003) investigou os efeitos de instruções sobre a falácia da conjunção. Em seu estudo, os participantes deveriam escolher entre estímulos simples correlacionados com diferentes probabilidades de ganhar reforço $(25,50 \mathrm{e}$ $75 \%$ ) e estímulos compostos com uma probabilidade que correspondia ao produto das probabilidades de seus constituintes (comportamento não-verbal). Ao longo das condições experimentais, as instruções tornavam-se progressivamente mais informativas de modo que, na última condição, era dito que para ganhar reforços com o composto era necessário ganhar reforços simultaneamente com cada um dos estímulos constituintes. Ao final do experimento, os participantes deveriam julgar os estímulos simples e os estímulos compostos em termos da probabilidade de ganhar reforços com cada um deles (comportamento verbal). Adicionar informações à instrução não afetou a escolha pelo composto, ou seja, a incidência da falácia, mas afetou os julgamentos de probabilidade, isto é, a magnitude da falácia. Esses resultados são consistentes com as conclusões de diversos estudos sobre controle verbal, os quais defendem a independência funcional entre o comportamento não-verbal e verbal (Paracampo, Souza, Matos \& Albuquerque, 2001; Rosenfarb, Newland, Brannon \& Howey, 1992).

A correspondência verbal-não-verbal foi também investigada por Abreu-Rodrigues e cols. (2005). Nesse estudo, os autores investigaram se a escolha por estímulos compostos seria afetada por manipulações na acurácia das estimativas. Na Fase de Treino, os participantes foram expostos a dois estímulos coloridos correlacionados com alta ou baixa probabilidade de reforço. Algumas vezes o experimentador solicitava ao participante que estimasse suas chances de ganhar pontos quando cada um dos estímulos era apresentado. Para o Grupo Acurado, o participante ganhava o máximo de pontos possível quando as probabilidades 0,8 e 0,2 eram correlacionadas com os estímulos de alta e baixa probabilidade, respectivamente. Para o Grupo Inacurado, ocorria o inverso, ou seja, o máximo de pontos era fornecido quando as probabilidades 0,8 e 0,2 eram apresentadas para os estímulos de baixa e alta probabilidade, respectivamente. E para o Grupo Incontrolável, a atribuição de pontos era independente da estimativa. Na Fase de Teste, durante a qual os participantes deveriam escolher entre estímulos compostos e simples, foi observado que as escolhas foram determinadas pela modelagem das estimativas e não pelas probabilidades programadas na fase anterior. $\mathrm{O}$ Grupo Acurado preferiu o composto quando comparado com o estímulo simples com baixa probabilidade, mas não quando comparado com o estímulo simples de alta probabilidade. Um resultado oposto foi obtido para o Grupo Inacurado. E, para o Grupo Incontrolável, não foi observada preferência por nenhuma das alternativas de escolha. Esses resultados indicam que as escolhas pelos compostos foram afetadas pelas contingências verbais (probabilidades estimadas) em oposição às contingências não-verbais (probabilidades programadas) presentes na Fase de Treino.

\section{Variáveis de Contexto}

A literatura tem demonstrado que pequenas mudanças no contexto em que os eventos são apresentados podem gerar mudanças nas estimativas apresentadas pelos participantes. Teigen, Martinussen e Lund (1996b), por exemplo, argumentaram que a ocorrência de falácia diminuiria consideravelmente se os problemas fossem concretos, em vez de fictícios. No Experimento 1, os participantes receberam o problema da Linda (evento fictício). No Experimento 2, os participantes tinham que estimar a probabilidade da Noruega ganhar cada um dos seus três primeiros jogos na Copa do Mundo de Futebol, que se iniciaria em dois dias, e conjunções desses jogos (evento real). No problema da Linda, 90\% dos participantes cometeram a falácia e, no problema da Copa do Mundo, essa incidência diminuiu para aproximadamente $50 \%$. Entretanto, em um estudo em que os participantes deveriam estimar os resultados de um referendum sobre a entrada de três países na União Européia, um evento também real, a falácia foi observada para $80 \%$ dos participantes (Teigen \& cols., 1996a). Os autores sugeriram que a maior incidência da falácia no problema do referendum provavelmente ocorreu porque os participantes julgaram os resultados do referendum como sendo causalmente relacionados.

Wolford e cols. (1990) também investigaram as características do contexto. No Experimento 1, esses autores estavam interessados no grau de familiaridade do contexto. Para tanto, os participantes foram expostos ao problema da matrícula em disciplinas (muito familiar), da corrida de cavalos (familiar) ou da Linda (pouco familiar). No problema da matrícula, os participantes tinham que indicar, dentre três cartões de matrícula, qual deles correspondia à matrícula efetuada por um aluno cujas características foram descritas no enunciado do problema (as outras matrículas eram de outros alunos desconhecidos). O primeiro cartão continha uma disciplina bastante representativa da descrição do aluno, o segundo continha uma disciplina pouco representativa e o terceiro continha essas duas disciplinas. No problema da corrida de cavalos, depois de descritas as características de dois cavalos que iriam participar de uma corrida, o participante deveria estimar a probabilidade de três resultados possíveis: o animal favorito ganhar, um outro animal chegar em segundo lugar e, por último, o favorito ganhar e o outro chegar em segundo lugar.

Os dados mostraram que o problema da matrícula gerou uma maior incidência de falácia $(98 \%)$ do que o problema da Linda (77\%), que, por sua vez, gerou mais falácia do que o problema da corrida de cavalos (52\%), não sendo, portanto, observada uma relação inversa entre grau de familiaridade com o contexto e incidência da falácia. Mas, por que o problema da matrícula gerou mais falácia do que o problema da corrida de cavalos? Para os autores, no problema da matrícula, julgar o composto como mais provável não seria uma falácia porque o contexto, embora familiar, já havia ocorrido e, assim, uma das matrículas necessariamente era do aluno. Não haveria violação da regra da conjunção, portanto, pois a alternativa com apenas uma disciplina não incluía a alternativa com as duas disciplinas, uma vez que a primeira representava a matrícula de uma outra pessoa. No problema da corrida de cavalos, por outro lado, como o contexto ainda 
não havia ocorrido, não era possível saber se o resultado da corrida corresponderia, de fato, a uma das três alternativas. Nesse caso, os limites impostos pela regra da conjunção deveriam ser respeitados, pois a alternativa com apenas um resultado continha a alternativa com o resultado de menor probabilidade. Os dados parecem corroborar essa sugestão, uma vez que o problema da corrida de cavalos obteve a menor incidência de falácia.

Os autores concluíram que o conceito de falácia deve ser restrito àquelas situações em que a aplicação de regras de inclusão é possível. Nessas situações, avaliar o composto como mais provável que seus eventos constituintes é um erro de julgamento. Mas, se a noção de inclusão não é aplicável, dizer que o composto é mais provável não é um erro de julgamento (ver também Dulany \& Hilton, 1991; Fantino \& Stolarz-Fantino, 1991; Hertwig \& Gigerenzer; 1999).

\section{Conclusão}

A falácia da conjunção é um fenômeno inquestionavelmente robusto, sendo observada em aproximadamente $2 / 3$ dos participantes investigados, incluindo aqueles com treino em lógica. Esse fenômeno tem sido investigado prioritariamente por psicólogos cognitivistas, mas, ultimamente, tem atraído também a atenção de alguns analistas do comportamento (e.g., Abreu-Rodrigues \& cols., 2005; Fantino \& cols., 1997). Esses pesquisadores têm apontado diversos fatores determinantes da falácia, tais como a representatividade entre eventos e o uso de regras probabilísticas errôneas, além de variáveis verbais e históricas.

A relação entre representatividade e variáveis históricas, entretanto, tem sido negligenciada. Ao definir representatividade como similaridade entre eventos, por exemplo, os pesquisadores da área não têm considerado, explicitamente, que essa similaridade e, conseqüentemente, a estimativa da probabilidade do composto, são determinadas pela história de aprendizagem de cada indivíduo. Dessa forma, é importante que investigações futuras enfatizem a identificação e manipulação experimental de aspectos históricos relacionados com a representatividade entre eventos. Outras variáveis também requerem estudos adicionais, algumas porque não foram até então pesquisadas, outras porque têm gerado resultados inconclusivos. Dentre essas variáveis destacam-se as instruções, as auto-instruções, o status social dos eventos a serem julgados, a magnitude das probabilidades dos eventos constituintes, a familiaridade do contexto, etc.

Em suma, a literatura indica que os indivíduos tendem a julgar os eventos compostos como mais prováveis que seus eventos constituintes, o que consiste em uma violação da regra da conjunção. Esse erro tem suscitado inúmeras investigações, as quais têm demonstrado, em geral, que o mesmo é suscetível à influência de variáveis ambientais passadas e atuais.

\section{Referências}

Abreu-Rodrigues, J., Baumann, A. A. \& Souza, P. (2005, maio). Verbal relations and the conjunction fallacy. Palestra apresentada na $31^{\text {st }}$ Convention of the Association for Behavior Analysis, Chicago: USA.
Agnoli, F. \& Krantz, D. H. (1989). Suppressing natural heuristics by formal instruction: The case of the conjunction fallacy. Cognitive Psychology, 21, 515-550.

Ahn, W. K. \& Bailenson, J. (1996). Causal attribution as a search for underlying mechanisms: An explanation of the conjunction fallacy and the discounting principle. Cognitive Psychology, 31, 82-123.

Anderson, N. H. (1965). Averaging versus adding as a stimuluscombination rule in impression formation. Journal of Experimental Psychology, 70(4), 394-400.

Bonini, N., Tentori, K. \& Osherson, D. (2004). A different conjunction fallacy. Mind \& Language, 19(2), 199-210.

Crandall, C. S. \& Greenfield, B. (1986). Understanding the conjunction fallacy: A conjunction of effects? Social Cognition, 4(4), 408-419.

Dulany, D. E. \& Hilton, D. J. (1991). Conversational implicature, conscious representation, and the conjunction fallacy. Social Cognition, 9(1), 85-110.

Fabre, J. M., Caverni, J. P. \& Jungermann, H. (1995). Causality does influence conjunctive probability judgments if context and design allow for it. Organizational Behavior and Human Decision Processes, 63(1), 1-5.

Fantino, E., Kulik, J., Stolarz-Fantino, S. \& Wright, W. (1997). The conjunction fallacy: A test of averaging hypothesis. Psychonomic Bulletin \& Review, 4, 96-101

Fantino, E. \& Savastano, H. (1996). Humans' responses to novel stimulus compounds and the effects of training. Psychonomic Bulletin \& Review, 3(2), 204-207.

Fantino, E. \& Stolarz-Fantino (1991). Rational analysis and illogical inference. Behavioral \& Brain Sciences, 14(3), 494.

Fiedler, K. (1988). The dependence of the conjunction fallacy on subtle linguistic factors. Psychological Research, 50, 123129.

Fisk, J. E. \& Pidgeon, N. (1998). Conditional probabilities, potential surprise, and the conditional fallacy. The Quarterly Journal of Experimental Psychology, 51A(3), 655-681.

Freeman, T. J. \& Lattal, K. A. (1992). Stimulus control of behavioral history. Journal of the Experimental Analysis of Behavior, 57(1), 5-15.

Gavanski, I. \& Roskos-Ewoldsen, D. R. (1991). Representativeness and conjoint probability. Journal of Personality and Social Psychology, 61(2), 181-184.

Hertwig, R. \& Gigerenzer, G. (1999). The 'conjunction fallacy' revisited: How intelligent inferences look like reasoning errors. Journal of Behavioral Decision Making, 12(4), 275-304.

Leddo, J., Abelson, R. P. \& Gross, P. H. (1984). Conjunctive explanations: When two reasons are better than one. Journal of Personality and Social Psychology, 47(5), 933-943.

Massaro, D. W. (1994). A pattern recognition account of decision making. Memory \& Cognition 22(5), 616-627.

McClure, J., Lalljee, M., Jaspars, J. \& Abelson, R. P. (1989). Conjunctive explanations of success and failure: The effect of different types of causes. Journal of Personality and Social Psychology, 56(1), 19-26.

Mellers, B., Hertwig, R. \& Kahneman, D. (2001). Do frequency representations eliminate conjunction effects? Psychological Science, 12(4), 269-275.

Moutier, S. \& Houdé, O. (2003). Judgment under uncertainty and conjunction fallacy inhibition training. Thinking and Reasoning, 9(3), 185-201. 
Paracampo, C. C. P., Souza, D. G., Matos, M. A. \& Albuquerque, L. C. (2001). Efeitos de mudanças em contingências de reforço sobre o comportamento verbal e o não verbal. Acta Comportamentalia, 9(1), 31-55.

Rodrigues, L. C. (2005). Efeitos da história de reforçamento e de punição sobre a falácia da conjunção. Dissertação de Mestrado, Universidade de Brasília, Brasília.

Rosenfarb, I. S., Newland, C. M., Brannon, S. E. \& Howey, D. S. (1992). Effects of self-generated rules on the development of schedule-controlled behavior. Journal of the Experimental Analysis of Behavior, 58(1), 107-121.

Shafir, E. B., Smith, E. E. \& Osherson, D. N. (1990). Typicality and reasoning fallacies. Memory \& Cognition, 18(3), 229-239.

Shanteau, J. (1975). Averaging versus multiplying combination rules of inference judgment. Acta Psychologica, 39, 83-89.

Sloman, S. A., Over, D., Slovak, L. \& Stibel, J. M. (2003). Frequency illusions and other fallacies. Organizational Behavior and Human Decision Processes, 91, 296-309.

Stolarz-Fantino, S., Fantino, E. \& Kulik, J. (1996). The conjunction fallacy: Differential incidence as a function of descriptive frames and educational context. Contemporary Educational Psychology, 21, 208-218.

Stolarz-Fantino, S., Fantino, E., Zizzo, D. J. \& Wen, J. (2003). The conjunction effect: New evidence for robustness. American Journal of Psychology, 116(1), 15-34.

Teigen, K. H., Martinussen, M. \& Lund, T. (1996a). Conjunction errors in the prediction of referendum outcomes: Effects of attitude and realism. Acta Psychologica, 93, 91-105.
Teigen, K. H., Martinunssen, M. \& Lund, T. (1996b). Linda vs. World Cup: Conjunctive probabilities in three-event fictional and real-life predictions. Journal of Behavioral Decision Making, 9, 77-93.

Tversky, A. \& Kahneman, D. (1983). Extensional versus intuitive reasoning: The conjunction fallacy in probability judgment. Psychological Review, 90(4), 293-315.

Wanchisen, B. A. (1990). Forgetting the lessons of history. The Behavior Analyst, 13(1), 31-37.

Wells, G. (1985). The conjunction error and the representativeness heuristic. Social Cognition, 3(3), 266-279.

Wolford, G., Taylor, H. A. \& Beck, J. R. (1990). The conjunction fallacy? Memory \& Cognition, 18(1), 47-53.

Zizzo, D. J. (2003). Verbal and behavioral learning in a probability compounding task. Theory and Decision, 54, 287-314.

Zizzo, D. J., Stolarz-Fantino, S., Wen, J. \& Fantino, E. (2000). A violation of the monotonicity axiom: Experimental evidence on the conjunction fallacy. Journal of Economic Behavior \& Organization, 41, 263-276. 